\title{
Relación entre medidas neuropsicológicas y ecológicas de funcionamiento ejecutivo en Preescolares y su predicción del rendimiento matemático. Un Estudio Piloto
}

\section{Lorena Zorrilla-Silvestre ${ }^{1}$, María Jesús Presentación- Herrero $^{2}$, Jesús Gil-Gómez ${ }^{3}$.}

\author{
${ }^{1}$ Departamento de Educación, Universidad Jaume I, Castellón. \\ ${ }^{2}$ Departamento de Psicología evolutiva, educativa, social y metodología, \\ Universidad Jaume I, Castellón. \\ ${ }^{3}$ Departamento de Educación, Universidad Jaume I, Castellón.
}

\section{España}

Correspondencia: Lorena Zorrilla Silvestre. Facultad de Ciencias Humanas y Sociales, Departamento de Educación.Universidad Jaume I, Av. de Vicent Sos Baynat, s/n (Campus de Riu Sec) 12071 Castellón (España). E-mail: zorrilla@uji.es 


\section{Resumen}

Introducción. En este estudio se exploraron las variables de funcionamiento ejecutivo (FE) que permitieron evaluar el FE tanto en el hogar como en el colegio. El objetivo fue comparar los resultados obtenidos en las evaluaciones de estas funciones en niños de 5 a 6 años, y comprobar qué medida de estas variables predice mejor el rendimiento matemático.

Método. Participaron 66 alumnos de tercer curso de Educación Infantil a los que se les administraron pruebas de memoria verbal y viso-espacial, de inhibición, de estimación del rendimiento matemático y el Behavior Rating Inventory of Executive Function (BRIEF), llevando a cabo un estudio correlacional y descriptivo.

Resultados. Los resultados mostraron correlaciones entre la evaluación de los niños con pruebas neuropsicológicas y las valoraciones ecológicas de profesores y padres; y entre las valoraciones del BRIEF, en el dominio de metacognición.

Conclusión. La medida que mejor predijo el rendimiento matemático fue el BRIEF de profesores, especialmente en la memoria de trabajo.

Palabras Clave: Funcionamiento ejecutivo, BRIEF, pruebas neuropsicológicas, rendimiento matemático, educación infantil. 


\title{
The relation between neuropsychological and ecological measures of executive functioning in Preschoolers and its prediction of mathemat- ics performance. A pilot study
}

\begin{abstract}
Introduction. This study explored the variables of executive functioning (EF) that permitted the evaluation of EF both at home and at school. The objective was to compare the results of the evaluations of these functions in children aged 5 to 6 years, and see to what extent these variables best predicted mathematical performance.
\end{abstract}

Method. Sixty-six third year preschool children took part. They were given tests of verbal memory and visuospatial inhibition, estimation of mathematical performance and the Behavior Rating Inventory of Executive Function (BRIEF), by carrying out a correlational and descriptive study.

Results. The results showed correlations between the evaluation of children with neuropsychological tests and the ecological assessments of teachers and parents; and between the BRIEF valuations in the metacognitive domain.

Conclusion. The measurement that best predicted maths performance was the BRIEF by teachers, especially in working memory.

Keywords: Executive functions, BRIEF, neuropsychological tests, mathematics achievement, preschool education. 


\section{Introducción}

Las funciones ejecutivas (FE) se han definido como los procesos que asocian ideas, movimientos y acciones simples que orientan a la resolución de conductas complejas, con la finalidad de alcanzar un objetivo (Anderson, Jacobs y Anderson, 2008; Flores-Lázaro, Castillo-Preciado y Jiménez-Miramonte, 2014; Meltzer, 2013). En este sentido, diferentes autores Diamond, 2013; Korzeniowski, 2011; Luria, 1988; Stuss y Benson, 1986; Van De Voorde, Roeyers, Verté y Wiersema, 2010; Welsh y Pennington, 1988 consideran que las FE abarcan una serie de procesos cognitivos entre los que destacan la anticipación, elección de objetivos, planificación, selección de la conducta, autorregulación, autocontrol y uso de retroalimentación (feedback). Dicha capacidad incluye funciones de regulación o de gestión como la posibilidad de iniciar el comportamiento y regulación de las emociones, inhibir las acciones de los estímulos, seleccionar objetivos relevantes de tareas, planificar y organizar un medio para resolver problemas complejos, utilizar estrategias de resolución de problemas de manera flexible cuando sea necesario y evaluar el comportamiento.

En términos globales, las FE posibilitan el establecimiento de un pensamiento estructurado en actividades de muy diversa naturaleza. Son como una constelación de capacidades cognitivas destinadas a resolver situaciones no esperadas o cambiantes, y es posible agruparlas en una serie de dimensiones o componentes para su estudio (Bausela y Santos, 2006; Lezak, 1995; Stuss y Levine, 2000).

Las funciones ejecutivas están implicadas en el funcionamiento cognitivo y en el desempeño socio-emocional, requiriendo de una valoración adecuada tanto de las características del problema a resolver como de las consecuencias inmediatas, de mediano y de largo plazo, de la respuesta seleccionada. Si bien esto exige participación de los distintos sistemas sensoriales, las funciones ejecutivas se caracterizan por su independencia del input, es decir, coordinan y regulan las respuestas según lo que se desea alcanzar con independencia de la información que está disponible (Verdejo-García y Bechara, 2010). En esta línea, en su estudio Liberman, Giesbrecht y Müller (2007) establecen relaciones entre las FE y aspectos emocionales apartir de medidas ecológicas. Estos consideran que los niños con una buena habilidad inhibitoria y verbal regulan mejor sus emociones.

\section{Las FE en la Educación Infatil}

Tradicionalmente, se consideraba que las FE no podían ser evaluadas en etapas tem- 
pranas debido a su complejidad y desarrollo prolongado. No obstante, según Anderson (2002), es posible identificar el surgimiento y desarrollo de las FE en preescolares e incluso en bebés. Baker et al. (1996) mostraron que los niños de entre 4 y 8 años, mejoran progresivamente el número de movimientos que deben realizar para completar tareas gracias al incremento en la capacidad de formar representaciones mentales y su manipulación. Del mismo modo, García-Molina, Enseñat-Cantallops, Tirapu-Ustárroz y Roig-Rovira (2009) concluyen que los niños desarrollan sus FE durante sus primeros 5 años y su desarrollo influirá positiva o negativamente en su capacidad para enfrentarse a situaciones novedosas y adaptarse a los cambios en su dia a dia. Diversos estudios manifiestan que los niños entre $3 \frac{1}{2}$ y $4 \frac{1}{2}$ años presentan dificultades para guiar sus acciones inhibiendo la respuesta dominante aunque estos mejoran conforme avanza la edad, los niños de 6 años prácticamente no presentan dificultades para realizar la acción (Diamond, 2002; Gerstadt, Hong y Diamond, 1994). Estos hallazgos indican que en las edades entre los 3 y los 4 años existe un progreso del proceso inhibitorio tanto de respuestas dominantes cognitivas y motoras, como de respuestas de espera con contenido motivacional, y que en niños mayores de 4 años, prácticamente se encuentran establecidas estas habilidades, por lo que se ha considerado que el control inhibitorio puede ser un proceso que permite el desarrollo adecuado de otras FE (Barkley, 1997).

Otras investigaciones han evidenciado que durante la infancia existe una mejora importante en la capacidad de memoria de trabajo tanto en la modalidad visoespacial como auditivo verbal, que se extiende incluso más allá de los 6 y 7 años, por lo que su desarrollo es más tardío que otros procesos, tales como el control inhibitorio, con el cual se encuentra relacionado (Liberman, Giesbrecht y Muller, 2007). Lang y Perner (2002) llegaron a la conclusión que los preescolares de entre 3 y 5 años manifestaban una importante mejora en las actividades de cambio que requieren un mantenimiento activo de la información.

\section{Funcionamiento ejecutivo y Dificultad en el Aprendizaje de las Matemáticas (DAM)}

Algunas investigaciones longitudinales, en la línea de la presente investigación, han sido desarrolladas con el objetivo de estudiar la capacidad predictiva de las funciones ejecutivas sobre el logro matemático. Así por ejemplo, se han encontrado relaciones significativas entre medidas de planificación, control inhibitorio, flexibilidad cognitiva, control atencional o memoria de trabajo en el último curso de preescolar y el posterior rendimiento en matemáticas, y han destacado el papel que las dificultades en una o varias de estas funciones puede 
jugar en la etiología de las DAM (Bull y Scerif, 2001; Bull, Espy y Wiebe, 2008; Clark, Pritchard y Woodward, 2010; Rimm-Kaufman, Curby, Grimm Nathason y Brock, 2009).

Recientemente Toll, Van der Ven, Kroesbergen y Van Luit (2011) han encontrado que la función ejecutiva que más predice las DAM es la memoria de trabajo, seguida en importancia por la inhibición, mientras que no hallaron poder predictivo en la flexibilidad cognitiva. Lo más sorprendente es que la MT tiene un valor predictivo superior al que tenían las habilidades preparatorias para las matemáticas. Son muchos los estudios que han examinado la contribución de las FE en las DAM mediante pruebas neuropsicológicas estandarizadas, pero pocas se han propuesto evaluarlas de forma más ecológica comparando ambas medidas como se realiza en este estudio.

\section{Objetivos e hipótesis}

La razón por la que nos centramos en el estudio de las FE en Educación Infantil, concretamente planificación, MT e inhibición, es el hecho de que es en dicha etapa donde se empiezan a desarrollar funciones que posteriormente marcarán la etapa de primaria. Por ello, consideramos necesario tener una mejor visión de cómo se desarrollan y cómo se manifiestan éstas, tanto en el ámbito escolar como familiar, para así, poder mejorar sus condiciones de desarrollo. En este sentido, se han planteado los siguientes objetivos de estudio:

1. Comparar los resultados obtenidos en la evaluación neuropsicológica de las funciones ejecutivas en infantil, con las valoraciones realizadas por progenitores y profesorado.

2. Comparar los resultados del cuestionario BRIEF en sus dos versiones, para progenitores y para profesorado, y conocer las relaciones existentes entre ambas valoraciones.

3. Averiguar qué medida de las variables de FE (Inhibición, cambio, control emocional o conducta, iniciativa, planificación, MT, organización de materiales, monotorización) predice mejor el rendimiento matemático.

Por su parte, las hipótesis planteadas son:

Hipótesis 1. Habrá una correlación significativa entre los resultados de la evaluación neuropsicológica y la de los padres y profesores en las variable de FE (Inhibición, cambio, control emocional o conducta, iniciativa, planificación, MT, organización de materiales, monotorización). 
Hipótesis 2. Habrá una correlación significativa entre las valoraciones dadas por los docentes y por los progenitores.

Hipótesis 3. La medida de las variables de FE que mejor predice el rendimiento matemático será la de los docentes.

\section{Método}

\section{Participantes}

El estudio se ha realizado con una muestra de 66 niños preescolares, 37 niños y 29 niñas, de diferentes colegios de la provincia de Castellón (6 públicos y 1 concertado), seleccionando al azar 6 alumnos por aula a criterio de los tutores. Los criterios de exclusión que se aplicaron fueron un CI menor de 70 y mayor de 130, alumnado con necesidades educativas especiales o con indicios de presentarlas y una incorporación tardía al sistema educativo. La edad cronológica de los participantes oscilaba entre 5 y 6 años, ya que todos cursaban el tercer nivel de Educación Infantil (P5). La puntuación de CI equivalente de todos los participantes ha sido extraída a partir de una tabla de conversión (Sattler, 1982) de las puntuaciones directas obtenidas en la prueba de cubos y vocabulario del WPPSI-R.

\section{Instrumentos}

\section{Memoria verbal}

Subtest de dígitos de la escala de inteligencia de Weschler (1980). Consta de dos tareas: recuerdo directo y recuerdo inverso de dígitos. En la tarea de recuerdo directo el niño debe repetir en el mismo orden series de números que el evaluador lee de forma oral. En la tarea de recuerdo inverso el niño debe repetir en orden inverso la secuencia de números que el evaluador lee oralmente.

Test de Memoria de conteo (Case, Kurland, y Goldberg, 1982). Esta prueba consta de una serie de tarjetas con puntos azules y amarillos dispuestos de forma aleatoria. Consiste en contar el número de puntos azules, decirlos en voz alta y a continuación, tras contar una serie de tarjetas, recordar el número de puntos azules citados en el orden correcto.

\section{Memoria viso-espacial}

Odd-one out (Henry y MacLean, 2003). En esta tarea el sujeto observa una tarjeta con tres figuras presentadas en fila y debe identificar cuál es diferente. Al final de cada ensayo el 
niño debe recordar la localización ésta en orden correcto señalando con el dedo en una tarjeta con espacios en blanco su posición.

Test de laberintos (Porteus, 2003). Se le muestra al sujeto un laberinto con una ruta trazada en él y a continuación se le pide que trace la misma ruta en un laberinto en blanco idéntico al anterior.

\section{Control inhibitorio y/o impulsividad}

Stroop Sol-Luna (Musso, 2009). Consta de dos condiciones. En la condición A, se muestra a los niños una página con 30 imágenes de soles y lunas dispuestas al azar en filas y columnas. Los niños son instruidos para responder sol a las imágenes con soles y luna a las imágenes con lunas tan rápido como puedan (dentro de un plazo de 45 segundos). Inmediatamente después se presenta la condición $\mathrm{B}$, en la que se les pide que respondan rápidamente "sol" cuando el evaluador señala una luna, y viceversa.

Test de golpeteo (Batería NEPSY; Korkman, Kirk y Keny, 2007). Se presentan al sujeto dos situaciones: en la primera, congruente, debe hacer lo mismo que el examinador, cuando éste da uno o dos golpes en la mesa; en la segunda, incongruente, cuando el examinador da un golpe, el sujeto debe dar dos, y cuando da dos, el sujeto debe dar uno.

BRIEF (Behavior Rating Inventory of Executive Function, Giogia, Isquith, Guy y Kenworthy (1996)). Es una escala compuesta por dos cuestionarios, uno para padres y otro para docentes, diseñados para evaluar los aspectos conductuales del funcionamiento ejecutivo en el hogar y en la escuela, respectivamente. Cada cuestionario contiene 86 ítems que proporcionan una información global de la función ejecutiva a partir de dos dominios principales: índice de regulación comportamental compuesta por las escalas de inhibición, control emocional y cambio; e índice de metacognición compuesta por iniciativa, memoria de trabajo, organización de materiales, planificación y monitorización, así como una puntuación global de función ejecutiva.

\section{Medidas de CI}

Subtest del WPPSI-R (Wechsler Preschool and Primary Scale of Intelligence; Wechsler, 1967).

Subtest vocabulario. Mide la fluidez verbal y manejo de vocabulario y requiere que el sujeto diga el significado de 32 palabras de dificultad creciente. 
Subtest Cubos. El sujeto debe construir con cubos unos dibujos, de complejidad creciente. Evalúa la capacidad para analizar, sintetizar y reproducir dibujos geométricos abstractos.

\section{Evaluación del rendimiento matemático}

Cuestionario de estimación del rendimiento matemático en Educación Infantil. Se elaboró para la investigación a partir del curriculum de EI. Está compuesto por 23 ítems divididos en 4 factores: numeración, operaciones básicas, geometría y medidas de estimación de magnitudes, y otro ítem con la valoración del docente en cuanto al rendimiento matemático global del alumno.

\section{Procedimiento}

Una vez obtenidos los permisos de Conselleria de Educación, Cultura y Deporte y del Consejo Escolar de cada centro (España), se procedió a planificar la recogida de datos. El orden de administración de las pruebas fue idéntico en los siete centros, en igual franja horaria y en diferentes semanas. Se utilizaron las mismas pruebas e instrucciones de aplicación. Las pruebas de FE fueron administradas de manera individual por el experimentador en una sala del centro escolar donde no había distractores. Los cuestionarios del BRIEF fueron entregados a profesores y progenitores quienes los cumplimentaron con ayuda del experimentador cuando fue necesario. Una vez recogidos los datos se introdujeron en el programa estadístico y se realizaron los análisis.

\section{Análisis Estadístico}

La investigación responde a las características de un diseño de estudio correlacional y descriptivo, ya que lo que se pretende es mostrar el grado en que determinadas variables están relacionadas. En el diseño se ha incluido un análisis predictivo, con la finalidad de determinar cuál de todas las variables de funcionamiento ejecutivo predice mejor el rendimiento matemático.

El software utilizado para la realización de todos los análisis fue el paquete estadístico SPSS 19.0. En un primer momento, se realizaron análisis de correlación entre las puntuaciones directas de las pruebas de FE administradas a los sujetos con las valoraciones de los profesores, y análisis de correlación de las puntuaciones directas con las valoraciones de los progenitores. Posteriormente se calcularon correlaciones entre las valoraciones de los progenito- 
res y los profesores dadas en el BRIEF. Por último, se examinó qué medida de las variables de FE predice mejor el rendimiento matemático, a partir de análisis de regresión lineal entre los factores numeración y operaciones del cuestionario cumplimentado por el docente, el total de dichos factores y la valoración del maestro sobre el rendimiento en matemáticas con las pruebas neuropsicológicas, el BRIEF de maestros y de padres.

\section{Resultados}

Previamente se analizó la normalidad de las pruebas aritméticas con el Test de Kolmogorov-Smirnov. Por otro lado, se ha controlado la posible incidencia del sexo, la edad y el CI, no encontrando diferencias estadísticamente significativas en ninguna de las tareas asignadas a las mismas.

Relación entre los resultados obtenidos en la evaluación a los niños y las valoraciones de progenitores y profesores en cuanto a inhibición y MT

Existen correlaciones negativas estadísticamente significativas entre las puntuaciones directas de todas las pruebas neuropsicológicas con todos los factores de iniciativa, memoria de trabajo, planificación, monitorización y, por consiguiente, con el dominio de metacognición y con el índice global de la escala, valorados por el profesorado, siendo la memoria de trabajo la que alcanza valores más altos en dígitos inversos $(r=-0,505 ; p<.001)$. En el apartado progenitores, es también la memoria de trabajo la que correlaciona significativamente con el mayor número de pruebas, especialmente con dígitos inversos $(r=-0,335 ; p=$ 0,008). Por otro lado, se observa una correlación baja y negativa entre el test sol/luna y el factor control emocional ( $r=-0,293 ; p=0,23)$ valorado por el profesorado, no existiendo correlación entre las pruebas de inhibición y el factor de inhibición, valorado tanto por los profesores como por los progenitores. La tabla 1 refleja las correlaciones entre las funciones ejecutivas de memoria de trabajo verbal, memoria de trabajo visoespacial e inhibición evaluadas en los niños y las valoraciones realizadas de las mismas funciones por los profesores y los progenitores a través del BRIEF. 
Tabla 1.

Correlaciones entre las variables de FE evaluadas por pruebas neuropsicológicas con las evaluadas por los progenitores y profesores mediante el BRIEF

\begin{tabular}{|c|c|c|c|c|c|c|c|}
\hline & & Inhibicic & & $M T$ & & & \\
\hline & & $\begin{array}{c}\text { Tarea } \\
\text { sol/lun } \\
a\end{array}$ & $\begin{array}{l}\text { Test de } \\
\text { golpe- } \\
\text { teo }\end{array}$ & $\begin{array}{c}\text { Dígitos } \\
\text { Directos }\end{array}$ & $\begin{array}{l}\text { Dígitos } \\
\text { Inver- } \\
\text { sos }\end{array}$ & $\begin{array}{c}\text { Con- } \\
\text { teo }\end{array}$ & $\begin{array}{c}\text { Odd } \\
\text { One Out }\end{array}$ \\
\hline \multirow{7}{*}{$\begin{array}{l}5 \\
0 \\
2 \\
2 \\
2 \\
2\end{array}$} & $\begin{array}{l}\text { Control } \\
\text { emocional }\end{array}$ &,$- 293^{*}$ & - & - & - & - &,$- 300^{*}$ \\
\hline & Iniciativa & - & - &,$- 331^{* * *}$ &,$- 483^{* *}$ & $443^{* *}$ &,$- 290^{*}$ \\
\hline & $M T$ & - & - &,$- 360^{* *}$ &,$- 505^{* *}$ & $412^{* *}$ &,$- 361^{* *}$ \\
\hline & $\begin{array}{l}\text { Planifica- } \\
\text { ción orga- } \\
\text { nización }\end{array}$ & - & - &,$- 296^{*}$ &,$- 493^{* *}$ &, $331^{\text {** }}$ &,$- 300^{*}$ \\
\hline & $\begin{array}{l}\text { Monitoriza- } \\
\text { ción }\end{array}$ & - & - &,$- 280^{*}$ &,$- 369^{* *}$ &, $336^{* *}$ &,$- 356^{* *}$ \\
\hline & $\begin{array}{l}\text { Metacogni- } \\
\text { ción }\end{array}$ & - & - &,$- 321^{* *}$ &,$- 466^{* *}$ & $-^{-}, 357^{* *}$ &,$- 326^{* *}$ \\
\hline & $\begin{array}{l}\text { Índice glo- } \\
\text { bal com- } \\
\text { puesto }\end{array}$ & - & - &,$- 282^{*}$ &,$- 406^{* *}$ &,$- 297^{*}$ &,$- 300^{*}$ \\
\hline \multirow{4}{*}{ 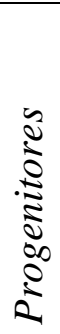 } & Cambio & - & - &,$- 271^{*}$ & - &,$- 261^{*}$ & - \\
\hline & $M T$ & &,$- 354^{* *}$ &,$- 272^{*}$ &,$- 335^{* *}$ & &,$- 256^{*}$ \\
\hline & $\begin{array}{l}\text { Planifica- } \\
\text { ción orga- } \\
\text { nización }\end{array}$ & - & - &,$- 305^{*}$ & - &,$- 297^{*}$ &,$- 261^{*}$ \\
\hline & $\begin{array}{l}\text { Metacogni- } \\
\text { ción }\end{array}$ & - & - &,$- 281^{*}$ & - & - & - \\
\hline
\end{tabular}

**. La correlación es significativa al nivel 0,01 (bilateral).

*. La correlación es significante al nivel 0,05 (bilateral).

Relación entre las valoraciones de progenitores y profesores mediante el cuestionario BRIEF

En el dominio de regulación de conducta existe una correlación media y significativa en el factor inhibición ( $r=0,371 ; p=0,004)$; mientras que en el domino de metacognición existe una correlación más alta y significativa entre las valoraciones realizadas por ambos, especialmente en memoria de trabajo $(r=0,576 ; p<.001)$. En la tabla 2 se presentan las correlaciones entre las valoraciones dadas en el BRIEF por los maestros y por los progenitores. 
Tabla 2.

Correlaciones entre las valoraciones de los progenitores y los profesores mediante el BRIEF
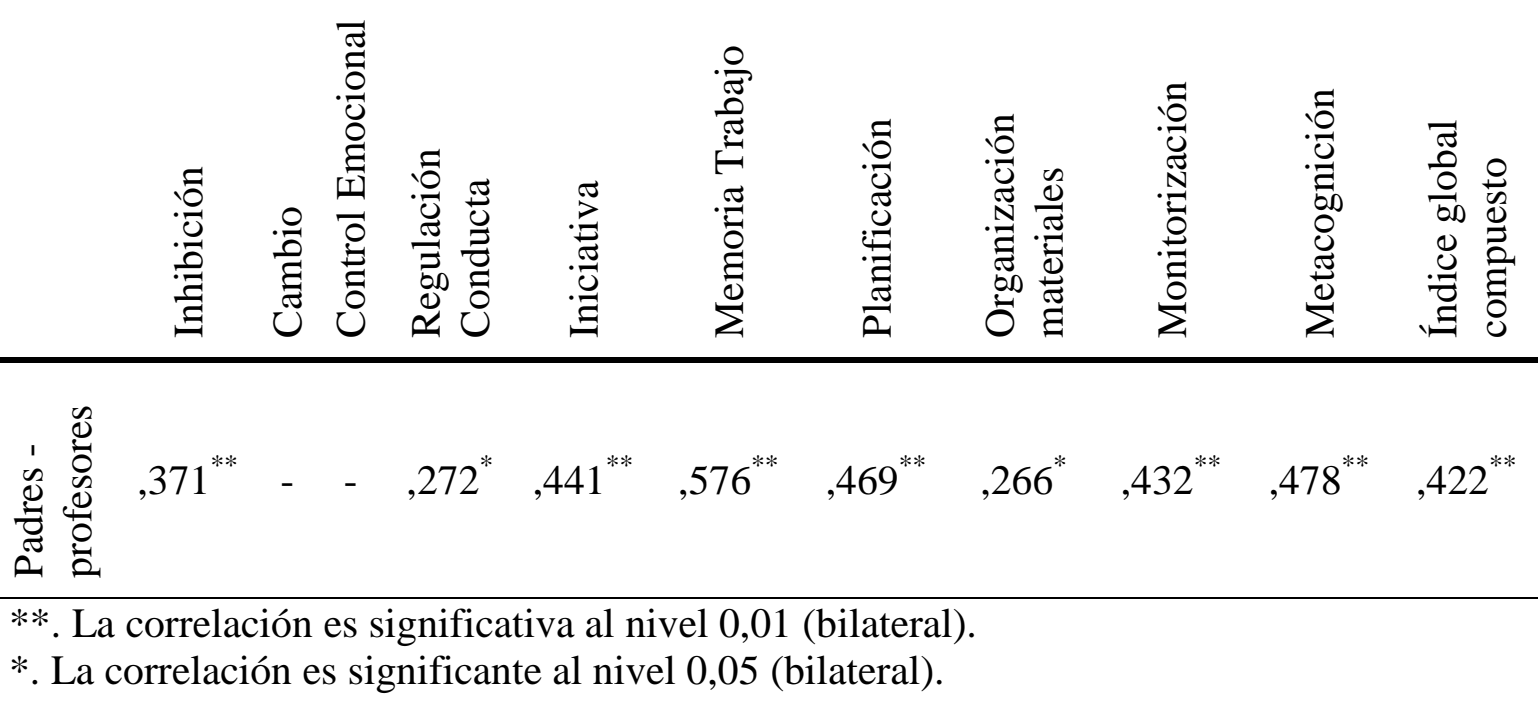

Predicción del rendimiento matemático según las medidas de las variables de FE

La tabla 3 recoge la contribución de las medidas de variables de FE a la predicción del rendimiento matemático, a partir de los resultados de los análisis de regresión lineal, realizados con los factores numeración y operaciones del cuestionario cumplimentado por los docentes y la valoración del maestro del rendimiento matemático del alumnado. En este caso se introdujo el CI en el primer bloque, y las medidas de las variables de FE en el segundo.

En todos los factores el CI fue significativo, explicado junto con otras variables. El factor numeración fue predicho por la prueba visoespacial $\left(\Delta \mathrm{R}^{2}=0,118 ; p=0,011\right)$ que junto con el CI $\left(\Delta \mathrm{R}^{2}=0,175 ; p=0,004\right)$ explicaban un $29,3 \%$ de la varianza total; por el CI, la MT y la organización de materiales valorados por el maestro que explican un 67,7\% de la varianza total; y la MT es el único factor valorado por los padres que explica un 29,4\% de la varianza.

La varianza total de las operaciones matemáticas estuvo explicada en un 50,8\% por pruebas de memoria de trabajo verbal como Dígitos inversos y conteo, en un 53,5\% por el CI y la MT según las valoraciones del maestro y en un 39,6\% por la MT e inhibición según la valoración de los padres. Finalmente, las pruebas de dígitos inversos, conteo y CI explican un $42,9 \%$ de la valoración que el maestro realiza del rendimiento matemático del alumnado, CI 
y la variable iniciativa valorada por el maestro predicen un $69,6 \%$ del factor, y CI junto a MT e inhibición del cuestionario de padres, un $56 \%$.

Tabla 3.

Resultados de los análisis de regresiones lineales de las variables de FE sobre el rendimiento matemático

\begin{tabular}{|c|c|c|c|c|c|}
\hline & & $\beta$ & $\overline{\mathrm{R}^{2}}$ & $\Delta \mathrm{R}^{2}$ & $p$ \\
\hline \multicolumn{6}{|l|}{ Factor Numeración } \\
\hline \multirow{5}{*}{$\begin{array}{r}\text { Pruebas neuro- } \\
\text { psicológicas } \\
\text { Brief-M }\end{array}$} & $C I$ & 0,185 & 0,175 & 0,175 & 0,004 \\
\hline & Odd One Out & 0,415 & 0,293 & 0,118 & 0,011 \\
\hline & $C I$ & 0,234 & 0,182 & 0,182 & 0,003 \\
\hline & $M T$ & $-0,477$ & 0,617 & 0,435 & 0,000 \\
\hline & $\begin{array}{l}\text { Organización } \\
\text { de materiales }\end{array}$ & $-0,313$ & 0,677 & 0,059 & 0,007 \\
\hline \multirow[t]{2}{*}{ Brief-P } & $C I$ & 0,303 & 0,172 & 0,172 & 0,005 \\
\hline & $M T$ & $-0,367$ & 0,294 & 0,122 & 0,010 \\
\hline \multicolumn{6}{|l|}{ Factor Operaciones } \\
\hline \multirow{3}{*}{$\begin{array}{l}\text { Pruebas neuro- } \\
\text { psicológicas }\end{array}$} & $C I$ & 0,229 & 0,224 & 0,224 & 0,001 \\
\hline & $\begin{array}{l}\text { Dígitos In- } \\
\text { versos }\end{array}$ & 0,372 & 0,433 & 0,209 & 0,000 \\
\hline & Conteo & 0,331 & 0,508 & 0,075 & 0,016 \\
\hline \multirow[t]{2}{*}{ Brief- $M$} & $C I$ & 0,299 & 0,234 & 0,234 & 0,000 \\
\hline & $M T$ & $-0,579$ & 0.535 & 0,301 & 0,000 \\
\hline \multirow[t]{3}{*}{ Brief-P } & $C I$ & 0,269 & 0,221 & 0,221 & 0,001 \\
\hline & $M T$ & $-0,538$ & 0,367 & 0,145 & 0,003 \\
\hline & Inhibición & 0,299 & 0,437 & 0,071 & 0,028 \\
\hline \multicolumn{6}{|l|}{ Valoración maestro } \\
\hline \multirow{3}{*}{$\begin{array}{r}\text { Pruebas neuropsico- } \\
\text { lógicas }\end{array}$} & $C I$ & 0,200 & 0,191 & 0,191 & 0,000 \\
\hline & $\begin{array}{l}\text { Dígitos In- } \\
\text { versos }\end{array}$ & 0,385 & 0,358 & 0,168 & 0,000 \\
\hline & Conteo & 0,295 & 0,429 & 0,070 & 0,010 \\
\hline \multirow[t]{2}{*}{ Brief-M } & $C I$ & 0,206 & 0,210 & 0,210 & 0,000 \\
\hline & Iniciativa & $-0,742$ & 0,696 & 0,487 & 0,000 \\
\hline \multirow[t]{3}{*}{ Brief-P } & $C I$ & 0,271 & 0,216 & 0,216 & 0,000 \\
\hline & $M T$ & $-0,682$ & 0,494 & 0,278 & 0,000 \\
\hline & inhibición & 0,291 & 0,560 & 0,060 & 0,005 \\
\hline
\end{tabular}

\section{Discusión y conclusiones}

El termino FE hace referencia a procesos y habilidades para llevar a cabo una conducta (Anderson, Jacobs, y Anderson, 2008; Flores-Lázaro, Castillo-Preciado, y JiménezMiramonte, 2014; Meltzer, 2013) compuesto por variables como la memoria de trabajo, la 
organización y planificación, la inhibición de respuestas, la flexibilidad cognitiva, la capacidad atencional o el control del propio estado emocional (Diamond, 2013; Korzeniowski, 2011; Van De Voorde, Roeyers, Verté, y Wiersema, 2010). Según autores como García, et al. (2013) y Van der Ven, Kroesbergen, Boom, y Leseman (2013) un mal funcionamiento de estas FE se relaciona con problemas en el pensamiento matemático, por ello nuestro estudio investiga la relación existente entre medidas neuropsicológicas y ecológicas de funcionamiento ejecutivo en infantil y que medida predice mejor el rendimiento matemático para poder actuar en consecuencia.

El primer objetivo del presente estudio fue determinar hasta qué punto existe una correlación significativa en la medición de las variables de funcionamiento ejecutivo mediante pruebas neuropsicológicas, y la valoración del maestro y del padre. Los resultados muestran que mientras no hubo relación entre las pruebas que median inhibición y el dominio de regulación conductual del BRIEF, la relación entre instrumentos que miden MT y dominio de metacognición fue significativa, especialmente entre las pruebas estandarizadas y las valoraciones de los maestros. Resultados que reproducen parcialmente los hallazgos de Dana Liebermann, Gerald F. Giesbrecht y Ulrich Müller (2007), en cuanto que no existe relación entre las valoraciones que proporcionan los padres en regulación emocional y las puntuaciones obtenidas con pruebas específicas que miden FE. Por otro lado, de acuerdo con informes anteriores, en las tareas de FE en niños en edad preescolar (Carlson, 2005; Gathercole, Pickering, Ambridge, y Wearing, 2004; Zelazo, Müller, Frye, y Marcovitch, 2003), las medidas de memoria de trabajo muestran tendencias significativas, tanto si se miden en un ambiente cotidiano como si se miden en laboratorio. Esto puede ser debido a que los comportamientos cotidianos relacionados con las FE y la regulación conductual pueden corresponder a procesos más generales de las FE, y no a aspectos más específicos de las mismas. Por ello, es difícil que los padres y maestros contesten a preguntan específicas de comportamiento a partir de impresiones generales.

El segundo objetivo de la investigación se centró en conocer las relaciones existentes entre las valoraciones de los profesores y de los padres sobre el funcionamiento ejecutivo de los niños. En relación al dominio de metacognición sí que existe correlación entre todos los factores, destacando una puntuación alta en la memoria de trabajo y planificación. Pero, por el contrario, en el dominio de regulación conductual no existe correlación debido a los factores cambio y control emocional. Lo que manifiesta que los discentes parecen regular mejor sus 
emociones en el contexto escolar que en el familiar. Por ello, se considera necesario analizar de forma complementaria la información aportada por observadores de diferentes contextos significativos para obtener un mejor conocimiento de las FE del niño; proponiendo que los docentes aporten directrices a los progenitores sobre cómo conseguir que sus hijos/as controlen y mejoren sus emociones en el contexto familiar.

A la luz de los resultados referentes al tercer objetivo, los análisis de regresión que se realizaron con las medidas de las variables de FE fueron relevantes. Todas las medidas de las variables junto con el CI contribuían a explicar el rendimiento de los factores matemáticos escogidos. Concretamente, la medida que más predecía cada factor era el BRIEF de maestros, seguido por el de padres y, sorprendentemente, las pruebas neuropsicológicas eran las que menos predecían las competencias matemáticas básicas. Estos datos indican que la clara predicción de una buena competencia matemática en niños de preescolar puede ser adquirida por múltiples medidas de fuentes independientes (Clark, Pritchard y Woodward, 2010).

El primer hallazgo a destacar es la correlación significativa entre la prueba de memoria de trabajo verbal, test de dígitos inversos, y la valoración del maestro y de los padres especialmente en MT, planificación y monitorización. Sin embargo, ninguna de las pruebas de inhibición correlacionó de forma significativa con el dominio de regulación conductual del BRIEF. Estos resultados confirman la validez de las pruebas ecológicas como el BRIEF para la medición de la metacognición, siendo más fiable la valoración del maestro. Otro hallazgo a destacar es la no correlación entre las valoraciones que el maestro proporciona sobre la regulación conductual y el control emocional del niño y las que proporcionan los padres. Esto puede ser debido a que los niños adoptan un comportamiento u otro dependiendo del contexto en el que se encuentran y de las normas establecidas en el mismo, creando diferentes percepciones de su comportamiento. Rechazando parcialmente la hipotesis establecida en el estudio en cuanto a que las valoraciones de los docentes y los progenitores coincidirian en todas las variables.

Y el último hallazgo a destacar es la importancia de la valoración del docente como predictor en el rendimiento matemático, tal y como se establecia en nuestra hipótesis. Los resultados muestran que la valoración del maestro es la que mejor predice el factor numeraciónel factor operaciones y la valoración total del rendimiento, incluso por encima de las pruebas neuropsicológicas estandarizadas. Por lo que, solamente pasando la parte del dominio de metacognición del BRIEF de maestros podemos predecir entre un 53,5\% y un 69,6\% del 
rendimiento matemático en los alumnos de infantil 5 años. No obstante, los hallazgos deben valorarse considerando varias limitaciones, como el reducido tamaño de la muestra y el hecho de que el cuestionario de estimación del rendimiento matemático en Educación Infantil fue cumplimentado por el profesorado sin un estudio piloto previo para analizar su validez y fiabilidad.

\section{Agradecimientos}

Este trabajo ha sido posible gracias a la subvención recibida del Ministerio de Economía y Competitividad de España para el proyecto titulado "Un estudio longitudinal de la contri-bución de DLM de las funciones ejecutivas, la motivación y las matemáticas habilidades básicas" (EDU2012-37452). En memoria de María Luisa Sanchiz Ruiz.

\section{Referencias}

Anderson, P. (2002). Assessment and development of executive function (EF) during childhood. Child Neuropsychology, 8(2), 71-82. doi:10.1076/chin.8.2.71.8724

Baker, S. C., Rogers, R. D., Owen, A. M., Frith, C. D., Dolan, R. J., Frackowiak, R. S. y Robbins, T.W. (1996). Neural systems engaged by planning: A PET study of the Tower of London task. Neuropsychologia, 34(6), 515-526.

Barkley, R.A. (1997). Behavioral inhibition, sustained attention, and executive functions: Constructing a unifying theory of ADHD. Psychological Bulletin, 121(1), 65-94. doi: http://dx.doi.org/10.1037/0033-2909.121.1.65

Bausela, E. y Santos, J.L. (2006). Disfunción ejecutiva: Sintomatología que acompaña a la lesión y/o disfunción del lóbulo frontal. Avances en salud mental relacional (ASMR). Revista online internacional, 5 (2). Recuperado de http://www.psiquiatria.com/revistas/index.php/asmr/article/view/901

Bechara, A., Damasio, H. y Damasio, A. R. (2000). Emotion, decision making and the orbitofrontal cortex. Cerebral Cortex, 10, 295-307. doi: 10.1093/cercor/10.3.295

Bull, R. y Scerif, G. (2001). Executive Functioning as a Predictor of Children's Mathematics Ability: Inhibition, Switching, and Working Memory. Developmental Neuropsychology, 19 (3), 273-293. doi:10.1207/S15326942DN1903_3

Bull, R., Espy, K. A. y Wiebe, S. (2008). Short-term memory, working memory and executive functioning: Longitudinal predictors of mathematics achievement at age 7. Developmental Neuropsychology, 33, 205-228. doi: 10.1080/87565640801982312 
Carlson, S. M. (2005). Developmentally sensitive measures of executive function in preschool children. Developmental Neuropsychology, 28, 595-616. doi:10.1207/s15326942dn2802_3

Clark, C. A. C., Pritchard, V. E. y Woodward, L. J. (2010). Preschool executive function predicts early mathematics achievement. Developmental Psychology, 46(5), 1176-1191. doi: $10.1037 / \mathrm{a} 0019672$.

Case, R., Kurland, M. y Goldberg, J. (1982). Operational efficiency and the growth of the short term memory. Journal of Experimental Child Psychology, 33(3), 386-404. doi:10.1016/0022-0965(82)90054-6

Diamond, A. (2002). Normal development of prefrontal cortex from birth to young adulthood: Cognitive functions, anatomy, and biochemistry. En D.T. Stuss, y R. T. Knight (Eds.), Principles of frontal lobe function, 466-503. Londres, UK: Oxford University Press.

Diamond, A. (2013). Executive Functions. Annual Review of Psychology, 64, 135-168. doi: 10.1146/annurev-psych-113011-143750

García-Molina, A., Enseñat-Cantallops, A., Tirapu-Ustárroz, B. y Roig-Rovira, T. (2009). Maduración de la corteza prefrontal y desarrollo de las funciones ejecutivas durante los primeros cinco años de vida, Revista de Neurologia, 48(8), 435-440. Recuperado de http://www.neurologia.com/pdf/Web/4808/bb080435.pdf

García, T., Rodríguez, C., González-Castro, P., Álvarez, D., Cueli, M. y González-Pienda, J. A. (2013). Funciones ejecutivas en niños y adolescentes con trastorno por déficit de atención con hiperactividad y dificultades lectoras. International Journal of Psychology and Psychological Therapy, 13(2), 179-194. Recuperado de http://www.ijpsy.com/volumen13/num2/354/funciones-ejecutivas-en-nios-yadolescentes-ES.pdf

Gathercole, S. E., Pickering, S. J. y Ambridge, B. (2004). The structure of working memory from 4 to 15 years of age. Developmental Psychology, 40, 177-190. doi: http://dx.doi.org/10.1037/0012-1649.40.2.177

Gerard A. Gioia, Ph.D., Peter K. Isquith, PhD, Steven C. Guy, PhD. y Peter K. Isquith, Ph.D. (1998). Behavior Rating Inventory of Executive Function, BRIEF. Florida: Psychological Assessment Resources, Inc.

Gerstadt, C. L., Hong, Y. J., y Diamond, A. (1994). The relationship between cognition and action: performance of children 3 1/2-7years old on a Stroop-like day-night test. Cognition, 53, 129-153. doi:10.1016/0010-0277(94)90068-X 
Henry, L., y MacLean, M. (2003). Relationships between working memory, expressive vocabulary and arithmetical reasoning in children with and without intellectual disabilities. Educational and Child Psychology, 20(3), 51-63. Recuperado de http://cogprints.org/5913/1/Educational_and_Child_Psychology_2003.pdf

Korkman, M., Kirk, U. y Kemp, S. (2007). NEPSY-Second Edition (NEPSY-II). San Antonio, TX: Harcourt Assessment.

Korzeniowski, C. G. (2011). Desarrollo evolutivo del funcionamiento ejecutivo y su relación con el aprendizaje escolar. Revista de Psicología, 7(13), 7- 26.

Lang, B. y Perner, J. (2002). Understanding of intention and false belief and the development of self-control. British Journal of Developmental Psychology, 20, 67-76. doi: $10.1348 / 026151002166325$

Lezak, M.D. (1995). Neuopsychological Assesment (3ºd.). New York: Oxford University Press.

Liebermann, D., Giesbrecht, G. F. y Muller, U. (2007). Cognitive and emotional aspects of self-regulation in preschoolers. Cognitive Development, 22(4), 511-529. doi:10.1016/j.cogdev.2007.08.005

Luria, A.R. (1988). El cerebro en acción (5 ed). Barcelona: Martínez Roca. p. 43-99.

Musso, M. (2009). Evaluación de funciones ejecutivas en niños: análisis y adaptación de pruebas en un contexto escolar. Revista Iberoamericana de Diagnóstico y Evaluación, 1(27), 157-178. Recuperado de http://www.aidep.org/03_ridep/R27/R278.pdf

Pickering, S. J., Baqués, J. y Gathercole, S. E. (1999). Bateria de Tests de Memòria de Treball. Barcelona: Laboratori de Memòria de la Universitat Autònoma de Barcelona. (Versión catalana de Pickering, S. \& Gathercole, S. (1997), Working Memory Battery. No comercializada).

Porteus, S.D. (2003). Test laberintos de Porteus. Madrid: TEA Ediciones

Rimm-Kaufman, S. E., Curby, T., Grimm, K., Nathanson, L. y Brock, L. (2009). The contribution of children's self-regulation and classroom quality to children's adaptive behaviors in the kindergarten classroom. Developmental Psychology, 45(4), 958-972. doi: $10.1037 / \mathrm{a} 0015861$.

Sattler, JM. (1982). Assessment of children intelligence and special habilities. (2 $2^{\mathrm{a}}$ ed.) Boston: Allyn \& Bacon.

Struss, D.T. y Benson D.F. (1986). The Frontal Lobes. New York: Raven Press.

Stuss, D. T. y Levine, B. (2000). Adult clinical neuropsychology: lessons from studies of the frontal lobes. Annual Review of Psychology, 53, 401-403. 
doi: 10.1146/annurev.psych.53.100901.135220

Toll, S.W.M., Van der Ven S.H.G, Kroesbergen, E.H. y Van Luit, J.E.H. (2011). Executive Functions as Predictors of Math Learning Disabilities. Journal of Learning Disabilities, 44(6), 521-532. doi:10.1177/0022219410387302

Van der Ven, S. H. G., Kroesbergen, E. H., Boom, J. y Leseman, P. P. M. (2011). The development of executive functions and early mathematics: A dynamic relationship. British Journal of Educational Psychology, 82, 100-119. doi: 10.1111/j.20448279.2011.02035.x

Van der Ven, S. H., Kroesbergen, E. H., Boom, J. y Leseman, P. P. (2013). The structure of executive functions in children: a closer examination of inhibition, shifting, and updating. British Journal of Developmental Psychology, 31(1), 70-87. doi: 10.1111/j.2044835X.2012.02079.X

Van De Voorde, S., Roeyers, H., Verté, S. y Wiersema, J. R. (2010). Working memory, response inhibition, and within-subject variability in children with attentiondeficit/hyperactivity disorder or reading disorder. Journal of Clinical and Experimental Neuropsychology, 32, 366-79. doi: 10.1080/13803390903066865

Verdejo-García, A. y Bechara, A. (2010). Neuropsicología de las funciones ejecutivas. Psicothema, $22 \quad$ (2), $227 \quad-235 . \quad$ Recuperado de http://www.psicothema.com/psicothema.asp?ID=3720

Welsh, M. C. y Pinnington, B.F. (1988). Assessing Frontal lobe functioning in children: Views from developmental psychology. Developmental Neuropsychology. 4(3), 199230. doi:10.1080/87565648809540405

Zelazo, P. D., Müller, U., Frye, D., y Marcovitch, S. (2003). The development of executive function in early childhood. Monographs of the Society for Research in Child Development, 68(3), vii-137. doi: 10.1111/j.1540-5834.2003.06803001.x 